\title{
Intrinsic dissolution testing: A tool for determining the effect of processing on dissolution behavior of the drug
}

Amjad Khan ${ }^{1,}{ }^{*}$, Zafar Iqbal2 ${ }^{2}$ Ismail Khan², Abad Khan ${ }^{3}$, Lateef Ahmad ${ }^{3}$, Muhammad Akhlaq Mughal ${ }^{4}$, Aman Ullah², Maria Kakar ${ }^{1}$

${ }^{1}$ Department of Pharmacy, Abasyn University, Peshawar, Pakistan

${ }^{2}$ Department of Pharmacy, University of Peshawar, Peshawar, Pakistan

${ }^{3}$ Department of Pharmacy, University of Swabi, Swabi, Pakistan

${ }^{4}$ Department of Pharmacy, Abasyn University, Islamabad, Pakistan

e-mail: amjadph@gmail.com

\section{ABSTRACT}

Intrinsic dissolution testing has been applied for characterization of pure API with respect to its dissolution behavior. The effect of formulation factors and processing parameters on dissolution rate is evaluated by dissolution testing of the final product, which is a time and resource-consuming process. Our objective was to use intrinsic dissolution testing for determining the effect of processing parameters on drug release. In the present study, an intrinsic dissolution testing method was developed for atenolol and validated according to the standard guidelines using USP-recommended dissolution media. Various experimental variables (compaction pressure and rotation speed of the disk) were optimized by studying at three levels. Atenolol was subjected to granulation in a super mixer granulator by applying standard wet granulation protocols. Granulation time and binder concentration were taken as process variables while dissolution rate was a response variable. Dissolution media had no interference with sample analysis, and all validation parameters for the developed method were in an acceptable range (\%RSD > 1). The intrinsic dissolution rate of atenolol $(1.84 \pm 0.13$ $\mathrm{mg} / \mathrm{cm}^{2} \mathrm{~min}$ ) was high due to its better solubility in dissolution media. The dissolution rate of atenolol was decreased by granulating with PVP under different conditions. We concluded that intrinsic dissolution testing method can be applied for determining the effect of processing parameters on dissolution rate of the API at the pre-compression level. This method will reduce experimentation for optimization of dissolution rate and will spare time and resources.

KEYWORDS: Atenolol, intrinsic dissolution testing, ICH Guidelines, method validation; dissolution

\section{INTRODUCTION}

he intrinsic dissolution rate (IDR) is the dissolution rate of a pure active pharmaceutical ingredient (API), where the conditions of surface area, temperature, agitation, $\mathrm{pH}$ of the medium and ionic strength are kept constant (1). APIs are commercially manufactured by different processes, resulting in variable characteristics like particle sizes, degree of hydration and crystalline forms. Intrinsic dissolution testing makes it possible to obtain data on the chemical purity and equivalence of the available APIs (2-4). It has been a key parameter during the development of new molecules, because the test can be executed with a small quantity and can predict potential dissolution problems $(2,5)$.

The IDR can be obtained by employing a specific device, where the compressed drug is exposed in a dissolution medium over a constant surface area and its value is expressed in $\mathrm{mg} / \mathrm{cm}^{2} \mathrm{~min}$. (6). USP recommends two methods for determining the IDR:

* Corresponding author.
- Rotating disk method: In rotating disk method, the powder compact is prepared and rotated in dissolution media at a specified rate.

- Stationary disk method: In this method, the disk is kept stationary in dissolution media and agitation is achieved by a paddle rotated at a specified speed.

Both the methods purport to maintain a constant surface area. The release from disk is referred to as a diffusioncontrolled process, thus any change in hydrodynamics caused by varying the rotation speed will influence the thickness of the diffusion layer and hence the dissolution rate (7). Some inherent problems with intrinsic dissolution testing are:

- Preparation of the disc by compression can produce changes in solid structure that may influence the dissolution behavior.

- The assumption of a constant surface area is valid only in the beginning of the determination. 
As the disk disintegrates or erodes, the surface area cannot be kept constant, so the disk should be compacted properly to prevent its erosion and disintegration. Furthermore, the test should be stopped when eroded particles are observed in dissolution media.

- Measurement of the dissolution rate on suspended materials does not normally include an assessment or measurement of inherent changes in surface area.

Intrinsic dissolution testing can be applied as an alternative for determining solubility, as it requires a small amount of material (8). Recent studies have proven its usefulness in the sphere of the Biopharmaceutics Classification System (BCS) because rather than equilibrium, intrinsic dissolution testing determines the rate and better correlation is expected (9). In a conventional solubility test, where a quantity of a drug is kept under constant agitation and temperature until the solution is saturated, any determination of the actual solubility of the material may be compromised because of the possible occurrences of recrystallization (10), leading to alteration of the crystalline form and hydrate or solvate formation.

During manufacturing of solid dosage forms (tablets/ capsules), APIs must be processed to improve rheological characteristics and mechanical strength to get the final product with uniform characteristics (11). Most of the APIs have poor flow and mechanical strength, which are improved by processing with other excipients, commonly by granulation technique (wet and dry granulation). Nature and level of processing can adversely affect dissolution characteristics of the product (12). The effect of processing parameters on drug release is evaluated by dissolution testing of the finalized dosage form, i.e. the processed API is converted to the dosage form (tablet/capsule) and evaluated for the drug release. It is a tedious job, involving further processing (converting the processed material to a dosage form), requiring resources and time, especially when desirable results are not achieved. To speed up the process of formulation development and to reduce the cost and experimentation, a reliable method for estimating the effect of processing parameters on dissolution rate was much needed. So, intrinsic dissolution testing has been extensively applied to evaluation of dissolution behavior of pure APIs in different crystalline states and dissolution media. No data are available regarding application of intrinsic dissolution testing in evaluating dissolution behavior of processed APIs. The objective of this study was to apply intrinsic dissolution testing for assessing the impact of processing parameters on the dissolution rate of the drug.

Atenolol is a $\beta$-adrenergic blocker, available in tablet dosage form in different strengths (25, 50 and $100 \mathrm{mg})$. Chemically, atenolol is 2-(4-\{2-hydroxy-3-[(propan-2-yl) amino] propoxy\} phenyl) acetamide, having a molecular weight of $266.311 \mathrm{~g} / \mathrm{mol}$. It is a class-III drug with good water solubility and poor permeability (13-15). Physically, atenolol is a white crystalline powder with poor flow and compressibility (16). Atenolol tablets are prepared by a wet granulation technique using polymeric binders.

In the present study, the intrinsic dissolution testing method was developed for atenolol and validated in accordance with the standard guidelines (USP and ICH guidelines) $(1,17)$. The developed method was used for determination of the intrinsic dissolution rate of pure atenolol and determination of the effect of processing on its dissolution behavior.

\section{MATERIALS AND METHODS}

\section{Materials and instrumentation}

Atenolol (purity 99.86\%; Wuhan Biocar Bio-Pharm Co., Ltd., China) was gifted by Ferozsons Laboratories Ltd. (Nowshera, Pakistan). The rest of the excipients, lactose (Molkerei Meggle, Germany), microcrystalline cellulose (FMC International, Ireland), polyvinyl pyrolidone (PVP k-30; ISP Technology, Texas) were purchased from local market of Peshawar, Pakistan. All the materials were pharmaceutical grade and used as received. Reagents (methanol, sodium 1-heptanesulfonate, dibasic sodium phosphate, dibutylamine, phosphoric acid) were purchased from the pharmaceutical market of Peshawar, Pakistan. All the reagents used in the study were of high performance liquid chromatography (HPLC) grade.

Granulation of atenolol was carried out using a laboratoryscale super mixer granulator, fluidized bed drier and cone mill (Yenchen, Taiwan). Dissolution studies were performed in a Pharma Test dissolution testing apparatus (Hainburg, Germany) while estimation of the drug content was carried out by a HPLC system (PerkinElmer Series 200, Norwalk, CT, USA). The HPLC system was equipped with an auto sampler, vacuum degasser, Peltier column oven, pump and UV-visible detector. The chromatographic data was analyzed on PerkinElmer TotalChrom workstation software (version 6.3.1) linked with the LC-system through PerkinElmer network chromatography interface $(\mathrm{NCl})$ 900. A hypersil ODS C-8 column $(250 \times 4.6 \mathrm{~mm}, 5 \mu \mathrm{m}$; Norwalk, USA) was used as a stationary phase and was protected by a PerkinElmer pre-column guard cartridge 
C18 $(30 \times 4.6 \mathrm{~mm}, 10 \mu \mathrm{m})$. Purified water was prepared by Milli-Q system (Millipore, Milford, MA, USA).

\section{Development of dissolution testing method}

The rotating disk method was developed for testing of IDR using dissolution media recommended by USP for dissolution testing of atenolol tablets (1). Powder was compacted into a nondisintegrating compact using a hydraulic press (PerkinElmer, USA) and subjected to dissolution testing. Compaction pressure and rotation speed of the disk are the two main parameters affecting the IDR of an API. The effect of both parameters on the IDR of atenolol was studied at three levels:

- Lower level of compaction pressure (1000 psi) and speed (50 rpm)

- Intermediate level of compaction pressure (2000

psi) and rotation speed (100 rpm)

- High level of compaction pressure (3000 psi) and rotation speed (150 rpm)

Criteria for selection of optimum experimental conditions were strength of the compact and dissolution rate.

\section{Validation of dissolution testing method}

According to the USP and ICH guidelines $(1,17)$, the dissolution method was validated for various parameters like linearity, specificity, accuracy, precision and stability.

\section{Linearity}

Linearity of the method was evaluated from the calibration curve constructed at seven different concentration points in the range of $50-250 \mu \mathrm{g} / \mathrm{mL}$. Calibration curve was constructed by plotting concentration of the solution versus area of the peak and slope (a); intercept (b) correlation coefficient $\left(r^{2}\right)$ and covariance (\%RSD) were determined from the regression analysis using the least squares method.

\section{Specificity}

Specificity of the dissolution method was evaluated by examining the effect of dissolution media on the peak characteristics (area, height, retention time and tailing factor). Dissolution media was evaluated without drug (blank) and with a known amount of drug, and the results were compared.

\section{Accuracy}

Percent recovery, calculated at three levels of concentration $(80 \%, 100 \%$ and $120 \%)$, was used for determining accuracy of the developed dissolution method. Percent recovery was calculated using following equation:

$$
\text { Percent recovery }=\frac{A}{B} \times 100 \text {------- Eq-1 }
$$

where

$$
\begin{aligned}
& A=\text { Peak area of the test solution } \\
& B=\text { Peak area of standard solution }
\end{aligned}
$$

\section{Precision}

Precision of the method was determined in terms of repeatability and intermediate precision. For repeatability, the dissolution test was performed in six vessels of dissolution testing apparatus under the same conditions. Intermediate precision was evaluated on the basis of intraday and interday studies. The intraday study was performed by repeating the dissolution test three times a day (at 8-h intervals) while the interday study was performed by repeating the dissolution test on daily basis (after each 24-h interval) for 3 consecutive days under the same conditions. Results were compared for variability. All analyses were performed in triplicate, and results were presented as mean $\pm \mathrm{SD}$; $\% \mathrm{RSD}$.

\section{Stability of atenolol solution}

Stability of the solution was determined at threeconditions (low temperature, $2-8^{\circ} \mathrm{C}$; ambient temperature, $24 \pm 3^{\circ} \mathrm{C}$; and elevated temperature, $40 \pm 3^{\circ} \mathrm{C}$ ) for 7 days. Samples were analyzed for drug content on a daily basis and percent recovery was calculated. At each sampling point, the analysis was carried out in triplicate. Results were presented as mean \pm SD.

\section{Determination of intrinsic dissolution rate}

The intrinsic dissolution rate of atenolol was determined according to the developed method. A nondisintegrating compact of $12 \mathrm{~mm}$ diameter was prepared by compacting sample (600 mg) under high pressure (2000 psi) with flatfaced round punches using a hydraulic press (PerkinElmer, USA). To minimize the effect of experimental errors, process variables related to the compact preparation (compaction, de compaction and ejection speed) were kept constant, throughout the study. The punch was lubricated using magnesium stearate before each compaction process.

Intrinsic dissolution testing was performed by rotating disk method at $100 \mathrm{rpm}$ in $0.1 \mathrm{~N}$ acetate buffer $\mathrm{pH} 4.6$ $(900 \mathrm{~mL})$, held at $37 \pm 2^{\circ} \mathrm{C}$ for $60 \mathrm{~min}$. Before starting the test, dissolution media was degassed and equilibrated to $37 \pm 2^{\circ} \mathrm{C}$. The disk along with holder were immersed in dissolution media and rotated at $100 \mathrm{rpm}$. Aliquots (3 $\mathrm{mL}$ ) were withdrawn at specified time intervals $(0,5,10$, $15,30,45$ and $60 \mathrm{~min}$ ) and analyzed for amount of drug 
released in triplicate $(n=3)$. After sampling, the volume of dissolution media was corrected with same volume held at the same temperature. A plot of the amount of drug released versus time was constructed, and the IDR was determined from the curve. Linearity of the curve was checked by regression analysis, performed using the method of least squares by Microsoft Excel, 2007 software. Adequacy of the fit was assessed from the regression coefficient $\left(R^{2}\right)$.

\section{Processing (granulation) of API and excipients}

In pharmaceutical manufacturing, wet granulation is the most efficient and commonly applied process for improving flow and compressibility of the material, especially when large doses of APIs with poor characteristics are to be processed (18). Processing of atenolol and excipients was carried out by standard wet granulation protocol, consisting of;

- Homogenization of the dry powder

- Wet massing and wet granulation

- Drying of wet granules

- Sizing of dried granules

In the presented study, atenolol was granulated with other excipients (as per Table 1), using aqueous solution of polyvinyl pyrolidone (PVP k-30) as binder. All ingredients were weighed accurately using a digital balance (Precisa, Switzerland), then sifted through mesh with a pore size of $600 \mu \mathrm{m}$ and loaded to the mixing pan of a laboratory-scale super mixer granulator (Yenchen, Taiwan). The mixing pan was equipped with a horizontally rotating, three-blade main impeller and vertically rotating chopper. Atenolol was mixed with excipients with the impeller at high speed and the chopper at low speed. Binder solution (aqueous solution of PVP) was added through the hopper at the specified rate, and wet massing was carried out with the impeller at low speed and chopper at high speed for 3 $\mathrm{min}$. The wet mass was granulated for the specified time (Table 2) with both the impeller and chopper at high speed. Wet granules were collected in an FBD (fluidized bed drier) container and dried using a laboratory-scale fluidized bed drier (Yenchen, Taiwan). The temperature of hot air passing through the granules was set at $80 \pm$ $5^{\circ} \mathrm{C}$, preheating time was $2 \mathrm{~min}$ and pause time and filter bag shaking time were $3 \mathrm{~min}$ and $20 \mathrm{~s}$, respectively. The granules were dried for the specified time $(25 \mathrm{~min}$ ). The dried mass was screened through a 2-mm mesh at $800 \mathrm{rpm}$, using a cone mill (Yenchen, Taiwan). The dried granules were collected in an air-tight container and stored until further use.
Table 1. Composition of Atenolol Granules

\begin{tabular}{|l|c|}
\hline Ingredients & Quantity (\%w/w) \\
\hline Atenolol & 50.00 \\
\hline Micro crystalline cellulose & 20.00 \\
\hline Lactose* & $23.0 / 18.0 / 13.0$ \\
\hline Primogel & 2.00 \\
\hline Polyvinyl pyrolidone (PVP k-30)* & $5.0 / 10.0 / 15.0$ \\
\hline Purified water & Quantity Sufficient \\
\hline
\end{tabular}

*PVP was used in three different concentrations and corrections were made in quantity of lactose, accordingly.

Table 2. Experimental Conditions for Processing of Atenolol Granules, Prepared by Wet Granulation Technique

\begin{tabular}{|l|c|c|}
\hline Trial No. & Granulation Time (s) & $\begin{array}{c}\text { Binder } \\
\text { Concentration* }\end{array}$ \\
\hline Trial 1 & 60 & $15 \% \mathrm{w} / \mathrm{w}$ \\
\hline Trial 2 & 60 & $10 \% \mathrm{w} / \mathrm{w}$ \\
\hline Trial 3 & 60 & $5 \% \mathrm{w} / \mathrm{w}$ \\
\hline Trial 4 & 45 & $15 \% \mathrm{w} / \mathrm{w}$ \\
\hline Trial 5 & 45 & $10 \% \mathrm{w} / \mathrm{w}$ \\
\hline Trial 6 & 45 & $5 \% \mathrm{w} / \mathrm{w}$ \\
\hline Trial 7 & 30 & $15 \% \mathrm{w} / \mathrm{w}$ \\
\hline Trial 8 & 30 & $10 \% \mathrm{w} / \mathrm{w}$ \\
\hline Trial 9 & 30 & $5 \% \mathrm{w} / \mathrm{w}$ \\
\hline
\end{tabular}

* Binder concentration is given as a percentage of the total weight of the granules.

Along with the operational parameters of the super mixer granulator (speed of impeller and chopper), concentration of binder solution and granulation time are the two main parameters governing the characteristics of granules and are usually optimized. In the present work, operational parameters of super mixer granulator (speed of the impeller and chopper) were kept constant while granulation time and binder concentration were taken as process variables, studied at three levels (high, middle and low) as defined in Table 2. Release rate of atenolol from granules was taken as a response variable.

\section{Dissolution testing of the processed granules}

The dissolution rate of atenolol from the processed granules was determined by applying the method developed for determination of the IDR, as described earlier in the manuscript. The dissolution media and experimental conditions were kept constant, and the dissolution rate was calculated under the same protocols.

\section{Analysis of dissolution samples}

During dissolution studies, samples were collected at specified time intervals, filtered and analyzed for drug released using HPLC. An Hypersil ODS C-8 column (250 
x $4.6 \mathrm{~mm}, 5 \mu \mathrm{m}$; Norwalk, USA) was used as a stationary phase and was protected by a PerkinElmer precolumn guard cartridge C $18(3 \times 4.6 \mathrm{~mm}, 10 \mu \mathrm{m})$. The mobile phase consisted of an aqueous phase $(700 \mathrm{~mL})$ and organic phase $(300 \mathrm{~mL})$. The aqueous phase was prepared by dissolving anhydrous dibasic sodium phosphate $(0.71$ $\mathrm{g}$ ) in an aqueous solution of sodium 1-heptanesulfonate (1.1 g in $700 \mathrm{~mL}$ of water). Dibutylamine $(2 \mathrm{~mL}$ ) was added, and $\mathrm{pH}$ was adjusted to 3 with phosphoric acid $(0.8 \mathrm{M})$. The organic phase (methanol, $300 \mathrm{~mL}$ ) was mixed with the aqueous phase, filtered through a $0.45 \mu \mathrm{m}$ filter paper and degassed. The mobile phase was pumped at a flow rate of $1.0 \mathrm{~mL} / \mathrm{min}$ at ambient temperature while the detector wavelength was set at $226 \mathrm{~nm}$. The atenolol solution (0.01 $\mathrm{mg} / \mathrm{mL}$ ) was prepared in the mobile phase was taken as the standard. Drug content of the sample was calculated by comparison of peak area of the sample and standard solutions containing the same concentration of atenolol. Each sample was analyzed in triplicate, and mean values were taken $(n=3)$.

\section{RESULTS AND DISCUSSION}

Atenolol is a crystalline powder, having low compressibility and poor rheological characteristics (15). It is formulated as a tablet dosage form in different strengths $(25,50$ and $100 \mathrm{mg} /$ tablet) and processed by wet granulation technique, using polymeric material as binder. In the presented work, an intrinsic dissolution testing method was developed, validated, and applied to determine the effect of processing parameters on the dissolution behavior of atenolol.

\section{Selection of optimum experimental conditions for dissolution testing}

Compaction pressure of the disk and speed of rotation are the two main parameters affecting the IDR of an API. Effect of both the parameters on intrinsic dissolution rate of atenolol was studied at three levels:

- Lower level of compaction pressure (1000 psi) and speed (50 rpm)

- Intermediate level of compaction pressure (2000 psi) and rotation speed (100 rpm)

- High level of compaction pressure (3000 psi) and rotation speed (150 rpm)

Differences in the IDR of atenolol at different levels of compaction pressure and rotation speeds were insignificant (less than 10\%). Because of better solubility of atenolol in dissolution media, the effect of experimental variables was insignificant, i.e. a harder disk rotated at a lower speed dissolved at almost the same rate as weakly compacted disk rotated at a higher speed. However, at lower compaction pressure the disk disintegrated (within $48 \mathrm{~min}$ ) and the test had to be stopped, as shown in Figure 1. Furthermore, at lower compaction the particles are not closely packed and there are chances of erosion of particles from the disk surface. Particle erosion from the disk surface was proven by variation in the values of IDR calculated at different points and higher SD among individual values. By increasing the compaction pressure, surface erosion and disintegration of the disk is prevented but the crystalline structure of API can change, which can adversely affect the results. So, the compaction pressure should be sufficient to prevent disintegration and surface erosion of the disk and should not affect the crystalline structure of API.

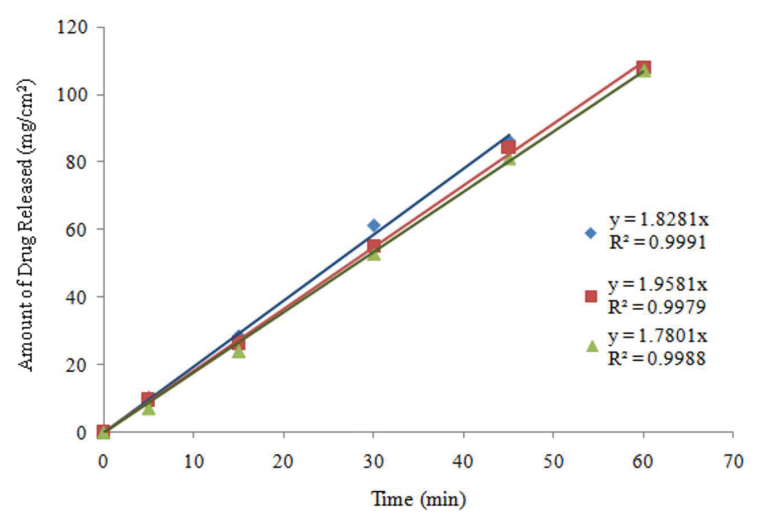

Figure 1. Intrinsic dissolution rate of atenolol, determined in $0.1 \mathrm{~N}$ acetate buffer $(900 \mathrm{~mL}$ ) at different experimental conditions; A) 1000 psi compression pressure and $50 \mathrm{rpm}$ rotation speed; B) 2000 psi compression pressure and $100 \mathrm{rpm}$ rotation speed; C) $3000 \mathrm{psi}$ compression pressure and $150 \mathrm{rpm}$ rotation speed.

Based on optimum disk strength (not disintegrating within $60 \mathrm{~min}$ ) and dissolution rate, a compaction pressure of $2000 \mathrm{psi}$ and rotation speed of $100 \mathrm{rpm}$ were selected as experimental conditions.

\section{Validation of dissolution testing method}

A rotating disk method was developed to determine the dissolution rate of pure and processed atenolol. The selected dissolution media consisted of $0.1 \mathrm{~N}$ acetate buffer $\mathrm{pH} 4.6(900 \mathrm{~mL})$, held at $37 \pm 2^{\circ} \mathrm{C}$ while the disk was compacted at $2000 \mathrm{psi}$ and rotated at $100 \mathrm{rpm}$. The developed dissolution testing method was validated according to the standard (USP and ICH) guidelines for various parameters like linearity, specificity, accuracy, precision and stability.

\section{Linearity}

Linearity of the method was determined from calibration curve, constructed at seven concentration levels in the 
range of 50 to $250 \mu \mathrm{g} / \mathrm{mL}$ using dissolution media as solvent. Regression analysis of calibration curve showed that the method is quite linear within the said concentration range. The regression equation and correlation coefficient values of the calibration curve are presented in Table 3.

Table 3. Validation Parameters of Intrinsic Dissolution Testing Method Developed for Atenolol

\begin{tabular}{|c|c|}
\hline Parameter & Result (Mean \pm SD; \%RSD) \\
\hline \multicolumn{2}{|l|}{ Linearity } \\
\hline Calibration Range & $50-250 \mu \mathrm{g} / \mathrm{mL}$ \\
\hline Regression Equation & $y=0.017 x+0.021$ \\
\hline Correlation Coefficient $(r)$ & 0.996 \\
\hline \multicolumn{2}{|l|}{ Accuracy } \\
\hline $80 \mathrm{mg}(80 \%)(\mathrm{n}=3)$ & $99.31 \pm 0.73 ; 0.74$ \\
\hline $100 \mathrm{mg}(100 \%)(\mathrm{n}=3)$ & $99.53 \pm 0.62 ; 0.62$ \\
\hline $120 \mathrm{mg}(120 \%)(\mathrm{n}=3)$ & $100.17 \pm 0.89 ; 0.89$ \\
\hline \multicolumn{2}{|l|}{ Precision } \\
\hline \multicolumn{2}{|l|}{ Repeatability } \\
\hline Vessel $1(V 1)(n=3)$ & $100.92 \pm 0.28 ; 0.28$ \\
\hline Vessel $2(V 2)(n=3)$ & $99.49 \pm 0.43 ; 0.43$ \\
\hline Vessel $3(V 3)(n=3)$ & $100.03 \pm 0.53 ; 0.53$ \\
\hline Vessel $4(V 4)(n=3)$ & $99.91 \pm 0.74 ; 0.74$ \\
\hline Vessel 5 (V5) (n=3) & $99.86 \pm 0.58 ; 0.58$ \\
\hline Vessel 6 (V6) $(n=3)$ & $100.01 \pm 0.51 ; 0.51$ \\
\hline \multicolumn{2}{|l|}{ Intermediate Precision } \\
\hline \multicolumn{2}{|c|}{ Intraday Reproducibility } \\
\hline $8 \mathrm{~h}(\mathrm{n}=3)$ & $99.64 \pm 0.36 ; 0.36$ \\
\hline $16 \mathrm{~h}(\mathrm{n}=3)$ & $99.55 \pm 0.67 ; 0.67$ \\
\hline $24 \mathrm{~h}(\mathrm{n}=3)$ & $99.95 \pm 0.49 ; 0.49$ \\
\hline \multicolumn{2}{|c|}{ Interday Reproducibility } \\
\hline Day $1(n=3)$ & $99.64 \pm 0.36 ; 0.36$ \\
\hline Day $2(n=3)$ & $99.83 \pm 0.69 ; 0.69$ \\
\hline Day $3(n=3)$ & $100.88 \pm 0.38 ; 0.38$ \\
\hline
\end{tabular}

Results are presented as mean $\pm S D$; $\% R S D$.

Dissolution media consisted of $0.1 \mathrm{~N}$ acetate buffer $\mathrm{pH}$ 4.6.

Experimental conditions: disk rotation speed, 100 rpm; disk compaction pressure, 2000 psi.

\section{Specificity}

Specificity of the method was estimated based on the chromatographic response of the blank dissolution media and dissolution media containing different concentrations of atenolol. The chromatogram of dissolution media was free of any interfering peaks, and different drug concentrations exhibited peaks with concentrationdependent area with constant retention time. Peak purity was further confirmed by examining through the peak purity tool provided in the HPLC software. The obtained value for the peak purity was $\approx 1$, indicating good peak purity.

\section{Accuracy}

Accuracy of the method was evaluated by percent recovery. Percent recovery from $95.0 \%-105.0 \%$ is recommended for the accuracy test. The percent recovery for atenolol was found to be in the range of $99.31 \%$ $100.17 \%$, as shown in Table 3, indicating good accuracy of the dissolution method.

\section{Precision}

Results for the intraday and interday precision are summarized in Table 3. The \%RSD value is less than $1 \%$, indicating good precision.

\section{Stability of atenolol solution}

Stability of atenolol was evaluated in dissolution media (0.1 N acetate buffer $\mathrm{pH} \mathrm{4.6)} \mathrm{and} \mathrm{compared} \mathrm{with} \mathrm{stock}$ solution (atenolol solution prepared in methanol). After completion of the test period ( 7 days), drug content of the samples was within the range of $99-100 \%$ (Fig. 2) of the initial concentration and no degradation product was observed in any of the chromatogram, indicating stability of atenolol in dissolution media. Similar results were obtained at ambient temperature $\left(24 \pm 3^{\circ} \mathrm{C}\right)$, refrigerator temperature $\left(2-8^{\circ} \mathrm{C}\right)$, and high temperature $\left(40 \pm 3^{\circ} \mathrm{C}\right)$, proving that dissolution media can be used without any stability issue.

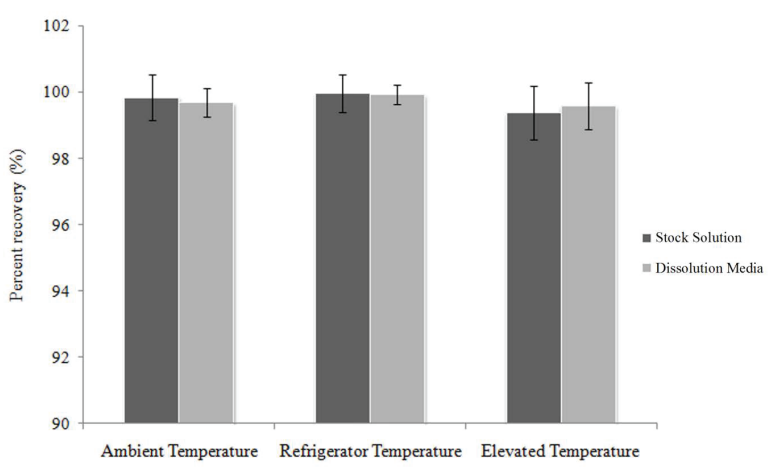

Figure 2. Percent recovery of atenolol samples prepared in methanol and dissolution media after storage at different temperatures for 7 days. Low temperature, $2-8^{\circ} \mathrm{C}$; ambient temperature, $24 \pm{ }^{\circ} \mathrm{C}$; elevated temperature, $40 \pm 3^{\circ} \mathrm{C}$

\section{Intrinsic dissolution rate of atenolol}

The IDR is the dissolution rate of the pure API when conditions such as surface area, temperature, agitation or stirring speed, $\mathrm{pH}$, and ionic strength of the dissolution medium are kept constant $(1,19)$. The IDR of atenolol was determined according to the developed method, using a rotating disk. The IDR of atenolol was fairly good $\left(1.84 \pm 0.13 \mathrm{mg} / \mathrm{cm}^{2} \mathrm{~min}\right)$ as shown in Figure 1 , due to its better solubility in dissolution media. The IDR was used as a standard for comparison with the dissolution rate 
of processed atenolol, as determined under the same conditions.

\section{Dissolution testing of the processed granules}

The dissolution rate of atenolol from the granules was studied by the method developed for determination of IDR under the same experimental conditions. A sample of granules ( $600 \mathrm{mg}$ ) was compacted under 2000 psi pressure and subjected to dissolution testing at $100 \mathrm{rpm}$ in $0.1 \mathrm{~N}$ acetate buffer $\mathrm{pH} 4.6(900 \mathrm{~mL})$. In the presented study granules of atenolol were prepared by wet granulation technique, taking binder concentration and granulation time as process variables. Various combinations of process variables resulted in different dissolution rate under the same experimental conditions. Increases in both the process variables increased hardness of the granules. The effect of binder concentration was more pronounced than granulation time and decreased dissolution rate in a concentration-dependent manner. During granulation, PVP forms strong bonds among the particles, holding them strongly and increases the inherent strength of the granules (20). Furthermore, PVP is a hydrophilic polymer which swells upon contact with water, forming a gel layer to control the drug release (21). When used in higher concentration, it forms a thick gel layer and retards drug release. In the present work, PVP was used as a binder in three different concentrations ( $5 \%, 10 \%$ and $15 \%$, w/w). A higher concentration of binder resulted in harder granules with higher inherent strength, having larger swelling time and a subsequently lower dissolution rate.

Higher binder concentration also resulted in harder compact. The cohesion index (crushing strength of the compact, compressed under maximum pressure without capping and lamination) was determined for granules (22), and it was found that an increase in binder concentration resulted in a higher cohesion index, indicating higher inherent strength of the granules. By increasing granulation time, hardness of the granules is increased, making it difficult for dissolution media to penetrate and dissolve the drug. At constant polymer concentration, reduction in granulation time increased dissolution rate and vice versa. Decrease in binder concentration lead to a higher dissolution rate. The highest dissolution rate $(1.50 \mathrm{mg} /$ $\mathrm{cm}^{2} \mathrm{~min}$ ) was observed with Trial 9, which had the lowest binder concentration $(5 \% \mathrm{w} / \mathrm{w})$ and smallest granulation time (30 sec). Similarly, the lowest dissolution rate $(0.94$ $\mathrm{mg} / \mathrm{cm} 2 \mathrm{~min}$ ) was shown by Trial 1, which had the highest binder concentration $(15 \% \mathrm{w} / \mathrm{w})$ and largest granulation time (60 sec), as shown in Figure 3. In comparison with the IDR of atenolol, a significantly decreased dissolution rate
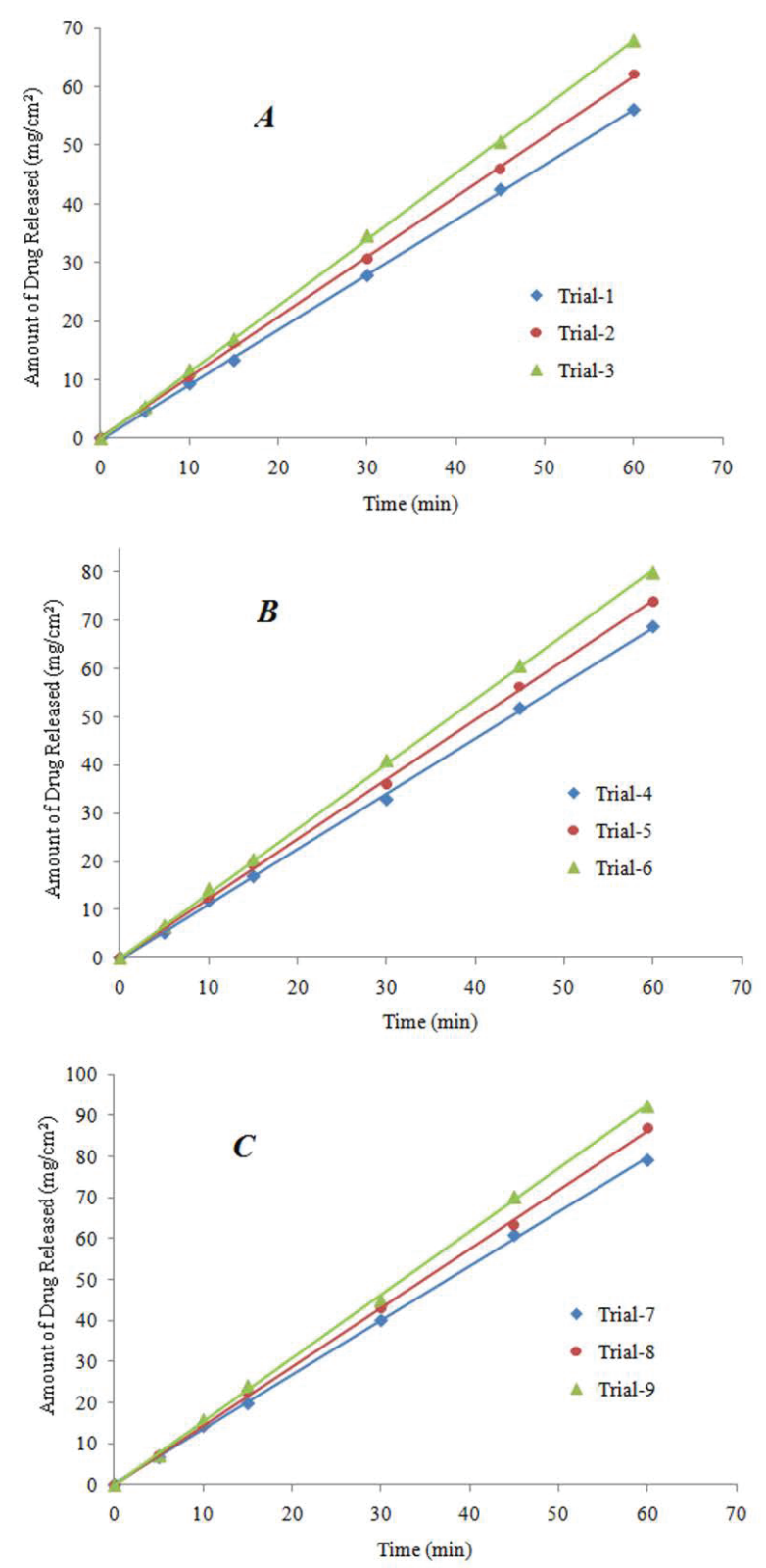

Figure 3. Dissolution rate of atenolol from granules processed under varying experimental conditions.

was observed with processed granules, as shown in Figure 4. The lowest dissolution rate (Trial 1) was $51.09 \%$ of the IDR while the highest dissolution rate (Trial 9) was $81.52 \%$.

\section{CONCLUSION}

An intrinsic dissolution testing method was developed for atenolol and applied to determine the dissolution rate of pure API and evaluate the effect of processing on dissolution rate. From the present study, we concluded that intrinsic dissolution testing can be applied to determine the dissolution rate of pure API and 


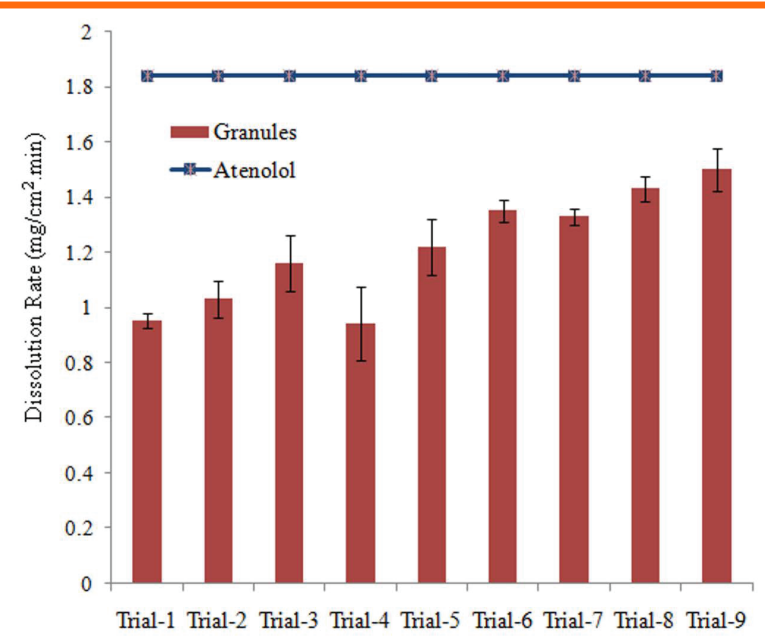

Figure 4. Comparison of intrinsic dissolution rate and dissolution rate of atenolol from processed granules.

processed APIs and to determine the effect of processing parameters. The effect of processing on dissolution rate is evaluated by compressing the granules to final dosage form, which is a time and resource-consuming process. The presented approach for determining the effect of processing on dissolution rate is rapid and cost effective. Intrinsic dissolution testing can determine minute changes in dissolution rate that are induced by processing. By properly developing and validating the dissolution testing method, the effect of processing on dissolution rate can be evaluated at the precompression level, which will shorten product development time and reduce cost and experimentation.

\section{ACKNOWLEDGEMENT}

We are thankful to the management of Ferozsons Laboratories Ltd., Nowshera, Pakistan, for providing facility to carry out the study.

\section{CONFLICT OF INTEREST}

The authors claim no conflict of interest.

\section{REFERENCES}

1. The United States Pharmacopeia and National Formulary USP38-NF-33. United States Pharmacopeial Convention, Inc.: Rockville, MD, 2015.

2. Zakeri-Milani, P.; Barzegar-Jalali, M.; Azimi, M.; Valizadeh, $\mathrm{H}$. Biopharmaceutical classification of drugs using intrinsic dissolution rate (IDR) and rat intestinal permeability. Eur. J. Pharm. Biopharm. 2009, 73 (1), 102-106. DOI: https://doi.org/10.1016/j. ejpb.2009.04.015.

3. Sehić, S.; Betz, G.; Hadžidedić, S.; El-Arini, S. K.; Leuenberger, $\mathrm{H}$. Investigation of intrinsic dissolution behavior of different carbamazepine samples. Int. J. Pharm. 2010, 386 (1-2), 77-90. DOI: https://doi.org/10.1016/j.ijpharm.2009.10.051.
4. Lehto, P.; Aaltonen, J.; Niemelä, P.; Rantanen, J.; Hirvonen, J.; Tanninen, V. P.; Peltonen, L. Simultaneous measurement of liquidphase and solid-phase transformation kinetics in rotating disc and channel flow cell dissolution devices. Int. J. Pharm. 2008, 363 (1), 66-72. DOI: https://doi.org/10.1016/j.ijpharm.2008.07.001.

5. Steele, G. Preformulation Predictions from Small Amounts of Compound as an Aid to Candidate Drug Selection. In Pharmaceutical Preformulation and Formulation: A Practical Guide from Candidate Drug Selection to Commercial Dosage Form; Gibson, M., Ed. Interpharm/CRC Press: Boca Raton, FL, 2004, 21-88.

6. Azarmi, S.; Roa, W.; Löbenberg, R. Current perspectives in dissolution testing of conventional and novel dosage forms. Int. J. Pharm. 2007, 328 (1), 12-21. DOI: https://doi.org/10.1016/j. ijpharm.2006.10.001.

7. Kulinowski, P.; Woyna-Orlewicz, K.; Rappen, G-M.; HaznarGarbacz, D.; Węglarz, W.P.; Dorożyńskib, P.P. An understanding of modified release matrix tablets behavior during drug dissolution as the key for prediction of pharmaceutical product performance - case study of multimodal characterization of quetiapine fumarate tablets, Int. J. Pharm. 2015, 484 (1-2), 235-245, DOI: http://dx.doi.org/10.1016/j.ijpharm.2015.02.040.

8. Sehić, S.; Betz, G.; Hadzidedić, S.; El-Arini, S.K.; Leuenberger, $\mathrm{H}$. Investigation of intrinsic dissolution behavior of different carbamazepine samples. Int. J. Pharm. 2010, 386 (1-2), 77-90. DOI: 10.1016/j.ijpharm.2009.10.051.

9. Yu, L. X.; Carlin, A. S.; Amidon, G. L.; Hussain, A. S. Feasibility studies of utilizing disk intrinsic dissolution rate to classify drugs. Int. J. Pharm. 2004, 270 (1-2), 221-227. DOI: https://doi. org/10.1016/j.ijpharm.2003.10.016.

10. Elder, D.; Holm, R. Aqueous solubility: Simple predictive methods (in silico, in vitro and bio-relevant approaches). Int. J. Pharm. 2013, 453 (1), 3-11. DOI: http://dx.doi.org/10.1016/j. ijpharm.2012.10.041.

11. Ansari, M.A.; Stepanek, F. The effect of granule microstructure on dissolution rate. Powder Technol. 2008, 181, 104-114. DOI: 10.1016/j.powtec.2006.12.012.

12. Horkovics-Kovats, S. Disintegration rate and properties of active pharmaceutical ingredient particles as determined from the dissolution time profile of a pharmaceutical formulation: An inverse problem. J. Pharm. Sci. 2014, 103 (2), 456-464. DOI: 10.1002/jps.23767.

13. Pezzini, B.R.; Grossl, A.D.; Muraro, A.; Zetola, M.; Brazzo, G.C.; Soares, L. Formulation and in vitro assessment of sustained release matrix tablets of atenolol containing Kollidon SR and carnauba wax. Afr. J. Pharm. Pharmacol. 2014, 8 (41), 1058-1065. DOI: 10.5897/AJPP2014.4126.

14. Usman, S.; Alam, A.; Nadeem, N.; Khawar, R.; Intesar, Z. Biowaiver Studies of Atenolol Tablets (100mg) - An Alternative to In Vivo Bioequivalence Studies. Nat. Prod. Chem. Res. 2014, 2 (1), 100125. DOI: 10.4172/ 2329-6836.1000125.

15. Kumare, M.M; Marathe, R.P.; Kawade, R.M.; Ghante, M.H.; 
Shendarkar, G.R. Design of fast dissolving tablets of atenolol using novel co-processed superdisintegrant, Asian. J. Pharm. Clin. Res. 2013, 6 (3), 81-85.

16. Gunjal, P. T.; Shinde, M. B.; Gharge, V. S.; Pimple, S. V.; Gurjar, M. K.; Shah, M. N. Design, development and optimization of $s(-)$ atenolol floating sustained release matrix tablets using surface response methodology. Indian J. Pharm. Sci. 2015, 77 (5), 563572. DOI: 10.4103/0250-474x.169036.

17. Note for Guidance on Validation of Analytical Procedures: Text and Methodology; CPMP/ICH/381/95, European Medicines Agency: London, 1995.

18. Li, J.; Tao, L.; Dali, M.; Buckley, D.; Gao, J.; Hubert, M. The effect of the physical states of binders on high shear wet granulation and granule properties: A mechanistic approach toward understanding high shear wet granulation process. Part II. Granulation and granule properties. J. Pharm. Sci. 2011, 100 (1), 294-310. DOI: https://doi.org/10.1002/jps.22261.

19. Grossjohann, C.; Eccles, K.S.; Maguire, A.R.; Lawrence, S.E.; Tajber, L.; Corrigan, O.I.; Healy, A.M. Characterization, solubility and intrinsic dissolution behavior of benzamide: dibenzyl sulfoxide cocrystal. Int. J. Pharm. 2012, 422 (1-2), 24-32. DOI: 10.1016/j. ijpharm.2011.10.016.
20. Dae Yeon, J.; Ji Eun, J.; Jeong Hwan, L.; Jae Won, Y.; Sang Mi, P.; Dongkwon, L.; Khang, G. Characterization and improvement of dissolution rate of solid dispersion of celecoxib in PVP K30/ Eudragit EPO. Polymer Korea 2014, 38 (4), 434-440. DOI: https:// doi.org/10.7317/pk.2014.38.4.434.

21. Khan, A; Iqbal, Z.; Khan, A.; Akhlaq Mughal, M.; Khan, A; Ullah, Z.; Khan, I. Modulation of $\mathrm{pH}$-independent release of a class II drug (domperidone) from a polymeric matrix using acidic excipients. Dissolution Technol. 2016, 23 (1), 32-40. DOI: dx.doi. org/10.14227/DT230116P32.

22. Khan, A.; Iqbal, Z.; Ibrahim, M.; Nasir, F.; Ullah, Z. Prediction of the effect of taste masking on disintegration behavior, mechanical strength and rheological characteristics of highly water soluble drug (itopride $\mathrm{HCl}$ ); an application of SeDeM-ODT expert system. Powder Technol. 2015, 284, 411-417. DOI: http://dx.doi. org/10.1016/j.powtec.2015.06.062. 